\title{
Analysis of Security of QoS in WiMAX and Mobile Networks
}

\author{
Adis Rahmanović ${ }^{1}$, Muzafer Saracević ${ }^{2}$ \\ ${ }^{1}$ University of Travnik, Faculty of Technical Studies, Bosnia and Herzegovina, \\ ${ }^{2}$ University of Novi Pazar, Department of computer sciences, Serbia
}

\begin{abstract}
In this paper we discuss several elements of importance for securing QoS in multimedia networks. Firstly, we present the first factor, which refers to understanding the characteristics of multimedia traffic in order to define and implement the QoS requirements. Secondly, factor refers to translation between QoS parameters that implies the distribution of system and network resources, and thirdly the factor establishes the appropriate QoS architecture that can provide the required QoS guarantees for multimedia applications.

We have been analyzing security-critical applications such as remote operation, which may require a guaranteed level of availability (hard QoS). There are basically two ways to secure a guaranteed QoS. The first is simply to provide a lot of resources, enough to meet the expected peak (peak) requirements with a significant security margin. This approach generously oversupplies the (over provisioning) network. We gave a detailed security analysis as features of WiMAX. More precisely, our analysis is based on the claim that its key feature of the WiMAX network is that the security layer is built into the protocol leg instead of being added later, i.e. the security layer is complex between PHY and MAC layers.
\end{abstract}

Keywords - WiMax, wireless network, Security, quality of service, mobile network.

DOI: $10.18421 /$ SAR33-06

https://doi.org/10.18421/SAR33-06

Corresponding author: Adis Rahmanović,

University of Travnik, Faculty of Technical Studies,

Bosnia and Herzegovina.

Email: adis.r@rmub.ba

Received: 06 June 2020.

Revised: 31 August 2020.

Accepted: 07 September 2020.

Published: 29 September 2020.

(cc)BY-NC-ND(C) 2020 Adis Rahmanović \& Muzafer Saracević; published by UIKTEN. This work is licensed under the Creative Commons AttributionNonCommercial-NoDerivs 3.0 License.

The article is published with Open Access at www.sarjournal.com

\section{Introduction}

WiMAX (Worldwide Interoperability for Microwave Access) is a technology aimed at wireless data access for long distances, and it is based on the IEEE 802.16 standard. During the previous research and implementation in the field of wireless telecommunications, we have come to the conclusion that WiMAX technology as well as its quality has great potential for application and the possibility for further research, development and application. This particularly applies to wireless broadband access. As there has been a significant increase in wireless communications over the past few years, quality of service (QoS) has become important to consider, primarily supporting various applications using network resources. This paper will examine general terms of service quality (QoS) in wireless networks. The IEEE 802.16 / WiMAX network architecture will be presented and the features of the MAC layer will be discussed, enabling the end-to-end service quality of the network service. Details on the number of services that are supported in WiMAX are discussed.[6] Through case studies, a special study will show and simulate VoIP traffic, with a special case study and simulation of multimedia streaming including analysis and conclusion, individually for special cases at first, and by comparing the obtained results from the two case studies and a joint conclusion. VOIP traffic is set that uses three supported service streams: Best Practice (BE), Real Time Reading Service (rtPS) and Non-Required Service (UGS) approval. Next, a case of using video streaming will be studied. Similar to the VoIP traffic case, the analysis is performed, which is based on four QoS parameters: bandwidth, average shake, average delay, and packet loss. Through our work, we will determine that WiMAX can offer a satisfactory quality of voice and video streaming services.

\section{Quality of Services (QoS) and Security}

Providing the required QoS is one of the important elements of designing and realizing multimedia 
networks. One of the QoS definitions that can be found in the literature is the following: "QoS represents a set of such qualitative and quantitative characteristics of a distributed multimedia system needed to realize the desired functionality of the application." The multimedia communications forum document under MMCF / 95-010 describes QoS requirements for applications, networks and services in multimedia telecommunications [5].

In this document, QoS is defined as "a set of service features that determine the degree of customer satisfaction provided by the telecommunication service". QoS, defined in this way, is determined by a set of service-related factors: providing service support, features of service operability, service access characteristics, and service integrity characteristics. In general, QoS is defined by a set of parameters that can be measured objectively. A set of relevant parameters, as well as the values of these parameters corresponding to the acceptable QoS, depend to a great extent on the type of multimedia application and its purpose.

Several elements of importance for securing QoS in multimedia networks can be distinguished:

1. Understanding the characteristics of multimedia traffic in order to define and implement the QoS requirements;

2. Translation between QoS parameters that implies the distribution of system and network resources;

3. Establish the appropriate QoS architecture that can provide the required QoS guarantees for multimedia applications.

The quality and efficiency of QoS management depend largely on the nature of multimedia traffic. Multidimensional modeling models are ranked according to appropriate groups; from those with constant bit rate (CBR) to those with variable bit rate (VBR). VBR traffic, which is characterized by compressed audio / video transmission, is sensitive to delays and has the "bursty" traffic characteristics. The differences between multimedia traffic and traffic in traditional networks are primarily related to:

1. Requirements for the transmission of continual media (audio and video transmission) in real time;

2. Significantly larger flows of individual media;

3. Distributed-oriented applications.

In traditional telecommunication networks, such as packet switched networks, QoS provision is on a qualitative basis, rather than providing services based on quantitative QoS requirements for each particular application. Real-time multimedia applications do not tolerate large variations in terms of delays and bandwidths. The constant increase in the number of multimedia applications required modification of existing network protocols to provide quantitative QoS parameters [4].

Real-time multimedia applications have characteristics that are significantly different from the features of standard network applications:

- Greater sensitivity to QoS parameters.

- Large number of multimedia applications in realtime generate continual traffic over a long period of time so that the network has to provide the necessary resources on a continual basis [4].

- The characteristics of multimedia traffic, i.e. its "inflexibility" and unpredictability, make it difficult to allocate the required resources. In the case of VBR traffic, allocation of resources based on average speed cannot provide QoS requirements, while allocation of resources based on maximum speed results in reduced network efficiency.

The complexity of the QoS problem is also reflected in the existence of differences between the QoS parameters related to the application and the network QoS parameters (e.g., bandwidth and bafer capacity). An important element in the analysis is the translation (mapping) of user / application QoS parameters into the network QoS parameters. Realizing this step is a complex task because of still incomplete knowledge of the user's perceptual characteristics. The transport system within the multimedia network is configured with network QoS parameters that describe the requirements regarding the quality of network infrastructure or network resources. Examples of network QoS parameters are flow (eg. packet size, bandwidth, "flashiness"), traffic characteristics (eg. packet loss, jitter, "end to end" delays) and performance characteristics (error correction, fragmentation, etc.). Troubleshooting QoS management in multimedia telecommunications is based on finding efficient network resource allocation, with QoS requirements and high resource utilization:

- Guaranteed QoS is usually obtained by analysis of so-called "Worst-case" traffic and by reserving the resources needed to secure the required QoS, even under the most unfavorable conditions in the network. This type of QoS performance, in general, has a low level of exploitation of resources due to resource isolation. Statistical QoS is, in the general case, realized by formal analysis of statistical traffic behavior, thus preventing QoS degradation below the determined deterministic level. As packages travel within a wireless network like WiMAX, they experience the following problems: 
- Delay: Unpredictable time for packets to reach the destination because of unavailability of network resources;

- Jitter: Source Packages will arrive at the destination with different delays;

- Out-of-order Delivery: When a set of connected packets are targeted over the Internet, different packages may take different routes, resulting in different delay times. The result is that the packets arrive in a different order than the one they were sent. This problem also requires special additional protocols that are responsible for redistributing the out-oforder packets after they arrive at their destination;

- Package Loss or Error: Sometimes the packages are incorrectly directed, damaged, or completely lost during the transfer. If the package is discharged, the receiver has to ask the sender to resend it. In the event of a major error detected in the bundle, the receiver has to detect the error and ask the sender to replay.

QoS in the area of telephony has been defined in 1994 by the International Telecommunication Union (ITU) under Recommendation E.800. This definition is very broad, citing six major components: support, operational, accessibility, availability, integrity and security. There are numerous parameters that can used for quantitative measurement, though only the name suggests that it is a qualitative measure of how reliable and consistent the network is. These are the bandwidth, delays in transmission or delays in the packet, the delay of shrinkage, the percentage of lost packages, and etc. When one uses different types of applications in the network it results in heterogeneous traffic loads. Traffic from a variety of applications may require a certain type of service quality, for example:

- Multimedia streaming may require guaranteed bandwidth.

- IP telephony (VoIP) may require stringent restrictions of distortion and delay.

- Video teleconferencing (VTC) requires low vibration.

- Emulation of a dedicated link requires guaranteed bandwidth and imposes limits for maximum delay and jitter.

- Security-critical applications, such as remote operation (health), may require a guaranteed level of availability (this is called hard QoS).

There are basically two ways to secure a guaranteed QoS. The first is simply to provide a lot of resources, enough to meet the expected peak (peak) requirements with a significant security margin. This approach generously oversupplies the (over provisioning) network. All packages get quality services enough to support QoS-sensitive applications. This approach is relatively straightforward, but most experts believe it is expensive to exercise in practice. This cannot be sustained if the peak demand rises faster than anticipated. It takes time to implement additional resources. For wireless networks, since the capacity of the wireless channel varies randomly over time, overseeing the network to provide QoS support will end up with the burden of resources. So, this approach is not feasible for commercial networks. The second approach requires people to make reservations and accept reservations only if routers are able to reliably operate them. This is known as the admission control. There are two methods popular for booting QoS support in the IP layer (layer 3):

\section{Integrated Services - Integrated Services (IntServ)}

\section{Differentiated Services (Diffserv).}

IEEE standard 802.16 includes the QoS mechanism in the MAC layer architecture (layer 2). The standard is designed to support a wide range of applications. These programs may require different levels of QoS. To meet these applications, standard 802.16 has defined five service flow classes.

Table 1. WiMAX 802.16 QoS model [7]

\begin{tabular}{|c|c|c|}
\hline & Description & Applications \\
\hline $\begin{array}{c}\text { Approved } \\
\text { services (UGS) }\end{array}$ & $\begin{array}{c}\text { For permanent } \\
\text { (CBR) and apps } \\
\text { depend on waiters } \\
\text { (delay) }\end{array}$ & VoIP \\
\hline $\begin{array}{l}\text { Real-time } \\
\text { calling service } \\
\text { (rtPS) }\end{array}$ & $\begin{array}{c}\text { For aplications of } \\
\text { variable bit rate and } \\
\text { depends on delay }\end{array}$ & $\begin{array}{l}\text { Audio } \\
\text { streaming, } \\
\text { video } \\
\text { streaming }\end{array}$ \\
\hline $\begin{array}{c}\text { Extended } \\
\text { Calling } \\
\text { Services in } \\
\text { Real Time } \\
\text { (ertPS) }\end{array}$ & $\begin{array}{c}\text { For aplications of } \\
\text { variable bit rate and } \\
\text { depends on delay }\end{array}$ & $\begin{array}{l}\text { VoIP with the } \\
\text { suppression of } \\
\text { silence }\end{array}$ \\
\hline $\begin{array}{c}\text { Calling service } \\
\text { is not real-time } \\
\text { (nrtPS) }\end{array}$ & $\begin{array}{c}\text { Variable rates and } \\
\text { non-real applications }\end{array}$ & FTP \\
\hline $\begin{array}{l}\text { The best effort } \\
\text { (BE) }\end{array}$ & The best effort & $\begin{array}{c}\text { E-mail, web } \\
\text { traffic }\end{array}$ \\
\hline
\end{tabular}

These service streams can be created, modified, or deleted by issuing Dynamic Service Addition (DSA), Dynamic Service Change (DSC) and Dynamic Service Deletion (DSD) messages. Each of these actions can be triggered by a subscriber station (SS) or a base station (BS), and they are executed through two or three "handshakes". The standard gives some rules for sorting Diffserv IP packets into different priority rows based on IPQoS index bits in the IP 
header. So, in principle, QoS architecture IEEE 802.16 supports both IntServ and Diffserv.

\section{WiMax Technology}

The name of the Worldwide Interoperability for Micro Access (WiMAX) technology was created by the WiMAX Forum, an organization set up in 2001 to promote the network implementation, compatibility and interoperability of the original standard based on the IEEE 802.16 workgroup. WiMAX technology operates across $10-66 \mathrm{GHz}$ frequencies on single carrier modulation, defined as wireless MAN-SC PHY, and frequencies below 11 $\mathrm{GHz}$ for non-line-of-sight (NLOS) optics; this option matches the standard wireless MAN-OFDM technology. IEEE standard 802.16 provides access to Fixed and Mobile subscribers in Optical Vision (LOS) configuration and no optical visibility (NLOS). Its main features include high bandwidth speed, high coverage, supported mobility, quality of service (QoS), and complete IP architecture. The original WiMAX standard, also known as Fixed WiMAX, provides a customizable end-to-end architecture that uses one carrier (SC), orthogonal frequency multiplexing (OFDM), and multiple access to orthogonal division (OFDMA)[6]. In theory, the IEEE 802.16 standard offers up to $50 \mathrm{~km}$ of bandwidth of $72 \mathrm{Mbps}$ throughput and up to $7 \mathrm{~km}$ without optical visibility (NLOS) in point-tomultipoint distribution. The connection between the base station (BS) and the mobile station (MS) is achieved through several command parameters and network functions used to handle upstream and downstream in mobile WiMAX. In 2005, IEEE standard 802.16e was released as an alternative and solution to the original standard for wireless mobility within WiMAX coverage [7].

WiMAX benefits include fast data transfer, low cost of access, open standards for wireless broadband services, eco-system preservation, low cost of subscription for mobile Internet and more. Hundreds of companies are participating in development and many companies have announced production plans for this technology. Only one WiMAX base station can cover the entire city including its wider environment. The main purpose of the system is to provide access to local networks, such as the Internet, video transmission, video telephony, IP telephony (IP-Internet Protocol) and the like. WiMAX can also show its advantages over broadband Internet access. On the one hand, it will enable the introduction of broadband internet access in the area where the construction of wire infrastructure is complicated or unprofitable, and on the other hand, it will allow competing operators to offer broadband Internet access at lower and higher speeds.
WiMAX is designed over the Physical Layer (PHY) and the Media Access Control (MAC) layer. This technology provides the ability to deliver quality of service (QoS) and security. WiMAX implementation is a subject to available spectrum resources, mobile WiMAX profiles are defined only for frequencies 2.3 to $2.4,2.5$ to 2.7 and 3.3 to $3.4 \mathrm{GHz}$, while the implementation of fixed WiMAX may range from 2 to $11 \mathrm{GHz}$ in the bandwidth. In 2005, IEEE released standard 802.16e or mobile WiMAX; this new standard has introduced new features such as mobility and portability, improved non-line-of-sight (NLOS) coverage through the AAS (Multi-Input Multi-Output Technology) technology, increased system gain and improved internal subcanning penetration [7]. This standard was created in the telecommunications industry as an alternative to the fixed and original IEEE 802.16 standard to support mobility between users. NonLine-of-sight (NLOS) propagation or full mobility is achieved in WiMAX technology thanks to the multiple access technique of orthogonal frequency division (OFDMA). This procedure consists of granting split to both subscription and uplink for several subscribers as a multiple access arrangement. This mechanism increases the number of end users given the fact that the radio frequency spectrum is used logically. In addition, power consumption is based on the distance from the base station, smaller power is transmitted to mobile users near the BS and greater power is available for MS away from the BS.

\subsection{WiMax Network Architecture}

The WiMAX architecture consists of three logical entities:

- Mobile station (MS) represents the end user or subscriber;

- Network Access Service (ASN), includes one or more base stations and an ASN gateway, and both belong to the radio access network entity;

- Connection Network (CSN), supports all IP network connectivity.

Each individual entity provides protocols and entire network access connectivity through certain elements such as base stations (BS), ASN entities, ASN-GW gateways, and external CSNs. These entities are linked through a reference point defined as R1, R2, R3, R4 and R5 in basic architecture. The reference points establish communication between the elements of the network over the air interface, and these reference points provide specific protocols and functions that define the entire architecture. The WiMAX Workgroup suggests the picture below, where multiple CSNs share the same ASN groups and vice versa. In this scenario, ASN and MS will 
exchange information so ASN can determine in which CSN any MS should be connected to provide access and connectivity. This CSN can be managed by the same operator or may belong to different operators.

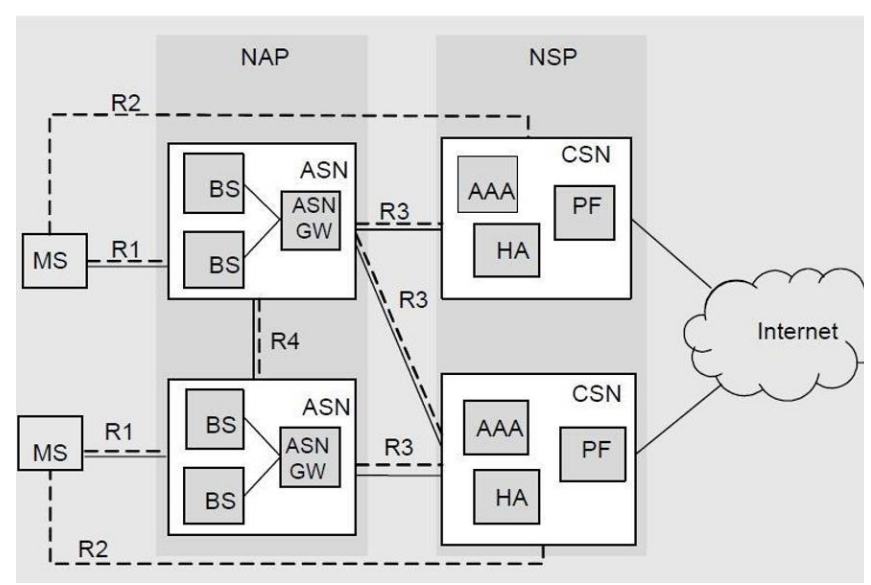

Figure 1. Multiple ASN Multiple CSN Models ${ }^{1}$

Mobile station (MS) or subscriber station (SS) are all users connected to the mobile network and this interface is defined in mobile WiMAX as a reference point R1, and it establishes a connection to the base station. ASN is a logical entity and enables mobile stations or users to access networks, within one ASN logically connect at least one Base Station (BS) and ASN Gateway (ASN-GW). The BS is logically linked to one or more ASN Gateways as illustrated in Figure 6 of the Workgroup for WiMAX. The Access Service Network (ASN) uses R1 Reference Points (RP) in MS and BS, R3 Network Services Interface (CSN), and R4 with other ASNs. The architecture made up of several ASN-GWs demands, which is a special way of defining mobility within ASN through the R4 interface, which is called inter-ASN mobility when the R3 interface does not exist. The ASN gateway represents the collection in the control plane segment that has a specific function with any ASN or CSN or any other ASN. The base station is a logical element within the ASN entity that establishes communication in the middle of MS and ASN. This entity includes the PHY and MAC layers specifications to ensure the corresponding function according to IEEE 802.16 standard. Usually, the base station represents three sectors with the assignment of one frequency, which provides scheduling functions for uplink and downlink resources. Physical implementation may include multiple base stations. OFDMA are OFDMs and serve to share main signal to bearers that are transmitted to avoid distortion and recover the original signal, if necessary, to the receiver side. OFDM is related to

\footnotetext{
1 "WiMAX End-to-End Network Systems Architecture - Stage 2: ArchitectureTenets, Reference Model and Reference Points," WiMAX Forum, December,2005, str. 29
}

point-to-point systems, while OFDMA provides true mobility and thus provides a unique interface for new technologies. Therefore, point-tomultipoint systems in mobile WiMAX architecture use OFDMA. Technically, the difference between OFDM and OFDMA is the way the binders are assigned to users. OFDMA has the ability to dynamically assign a subset of these subscribers to individual users, using either multiple access time division (TDMA) or multiple access frequency allocation (FDMA) for multiple users.

Duplex is a process in which the transmitter and receiver achieve bidirectional communication, and it is defined as semi-duplex mode and full duplex mode. In semi-duplex communication, transmission over the channel is a separate process, in other words, while the transmitter transmits the data, the receiver has to wait for receiving all data for the purpose of responding or starting a new transmission. The WiMAX system also supports both TDD and FDD on both half-duplex and duplex modes, labeled as H-FDD and F-FDD. However, the original edition of mobile WiMAX only takes into account the TDD mode. TDD's main features:

- TDD allows adaptation to the downlink / uplink ratio to effectively support asymmetric traffic;

- TDD guarantees reciprocity channels for better support for link adaptation;

- TDD requires only one channel for downlink and uplink, which offers greater efficiency.

In wireless spaces, the signal quality received from the mobile station is subject to loss of path, interference, weakness and noise. Therefore, the adaptive modulation and coding scheme (AMC) of mobile WiMAX technology has been introduced to mitigate these factors and improve network and capacity coverage. Therefore, the adaptive modulation and encoding scheme (AMC) not only improves capacity but also enables efficient use of permeable power by which data transfer rates achieve optimal performance and provide QoS between the base station and the mobile station. Technically, this process is also known as a downlink and uplink transfer adapter scheme, ranging from 64QAM to BPSK. The WiMAX Access Control Layer (MAC) protocol is designed for point-to-multipoint wireless broadband applications. This interface covers the need for a large amount of data both from the base station as a DL and to a base station such as UL. The MAC layer provides network functionality to the PHY layer and delivers features that deliver mobility, energy efficiency and quality of service (QoS) for mobile users. One of the most important features is the dynamic allocation of bandwidth which mitigated signal interference. Constant MAC convergence has the capacity to support any future IP 
protocol. The WiMAX MAC layer uses a system error mechanism and this requires automatic retransmission (ARQ), which works between MAC and PHY layers. This mechanism requires retransmission of the packets received with a fault, this process achieves high data transfer rates. Some of the features of WiMAX are:

- High data intensity: data transfer up to $63 \mathrm{Mbps}$ downlink and up to 39 Mbps in uplink can be achieved in WiMAX in $10 \mathrm{MHz}$ channel. This is possible because of the inclusion of MIMO (Multiple Input Multiple Output) antenna techniques with flexible sub-channel schemes, larger MAC frames, advanced coding and modulation.

- Scalability: WiMAX technology is designed to be able to scalability in various channels from 1.25 to $20 \mathrm{MHz}$ according to different requirements in the world.

- Security: Another key feature of the WiMAX network is that the security layer is built into the protocol leg instead of being added later. The security layer is complex between PHY and MAC layers. Messages for authentication and exchange of keys are defined as part of the MAC layer. The MAC layer performs encryption based on the keys agreed upon during the key exchange phase.

- Mobility: WiMAX supports optimized latency delivery schemes less than 50 milliseconds to ensure that real-time applications such as VoIP work without degradation of services. Flexible key management schemes ensure that security is maintained during handover.

QoS: Finally, the underlying assumption of the MAC architecture of IEEE 802.16 is QoS.

\subsection{QoS Factor in WiMAX and Mobile Networks}

Within the packet data network there are three main parameters that are key to network performance and WiMAX QoS, and these three parameters are:

- Latency: Latency is a measure of delays in the system;

- Jitter (jitter): the jitter is the variability of the packet latency over the network;

- Package Loss: Package Loss is the term used to denote packet data loss during network override.

The WiMAX standard offers four categories to prioritize traffic and thus improve QoS:

1. Unqualified Service Allowance (UGS);

2. Real Time Calling Services (rtPS); calling without actual time (NrtPS), and

3. Best attempt (BE).

Each service is intended for specific applications.

\subsection{Potential of Using WiMAX Networks}

WiMAX systems will offer basic infrastructure for smart cities design, where it is simultaneously processing an extremely large amount of data (large data) and sensitive real-time applications. WiMAX can be used for constant monitoring of bridges, remote city lighting control and traffic monitoring to achieve greater overall efficiency and safety [3]. The benefits of WiMAX over $4 \mathrm{G}$ and $5 \mathrm{G}$ networks are that WiMAX implementation is much cheaper than the $4 \mathrm{G}$ and $5 \mathrm{G}$ network implementation, and WiMAX is also an excellent choice for private mobile broadband wireless networks [1]. The biggest advantage of WiMAX at the time of development is rural and underdeveloped areas. This has become very popular in Australia and Africa, because large and sparsely populated areas can cover well enough without a widespread infrastructure that costs a lot of money and takes a lot of time to grow.

The application of WiMax in the region is different, so that the application of this technology has come to Montenegro and Serbia, where this technology is covered by larger cities with a tendency of further spread and premature rural environments. The situation in Croatia is such that only one company has started using WiMax infrastructure, although several of them have been granted concessions to implement it. The situation in $\mathrm{BiH}$ is the worst in this area, as apart from certain elaborate and declarative approaches to the implementation of this technology, there are obstacles due to the freedoms occupation by public broadcasters, because the digitization process of the television signal has not yet been completed. The potential for further intensive use of WiMax is the further development and application of ICT services.

\section{QoS for WiMax}

\subsection{Voice over IP}

VoIP is a set of technologies, methodologies, communication protocols and portable technologies that enable voice over IP communication. When establishing, maintaining, and deleting a communication connection or a call, VoIP uses various protocols, compression and encoding algorithms (codecs). In order for a VoIP call to be realized it is necessary to undertake a number of steps to enable it to be established. These steps are as follows: initial signaling, call setup, analogue-todigital voice conversion, encoding and compression, packing, transmission, decoding and decompression, and digital-to-analog signal conversion. VoIP uses a different communication standard that uses H.323 I Session Initiation Protocol (SIP) most widely.

Signalization protocols in the VoIP network need to: 
- Ensure the establishment, management and termination of the communication connection;

- Provide scalability (the VoIP network must be able to grow with the number of users and accept a large number of end user devices and accept a large number of calls simultaneously);

- Provide flexibility (VoIP network capability to introduce new functionality without the need for large-scale interventions and network changes);

- Ensure standardization (implies compatibility of devices and solutions of different manufacturers so that they can work together smoothly),

- Enable network management;

- Enable tariffing (billing of services).

VoIP service has a number of advantages: enhanced speech quality, greater availability, cost reduction, mobility, integration of different media, new services, real-time services, use of an existing computer network and improved utilization of the bandwidth. VoIP has built-in mechanisms for recognizing and suppressing silence, because at a time when one communicator tells stories, others listen, and in that case does not transmit any packets, which reduces the server load, it requires a lower bandwidth.

\subsection{Video Transmission}

The Mobile Image Experts Group has developed digital standards for video coding processes that coordinate the transfer of multiple forms of media (multimedia). Using H.264 / MPEG-4 AVC and hardware that enables H.264 compression, transportation, and decompression, telecommunications companies can raise their revenue with new interesting services such as videoon-demand (VOD), HDTV transmission and interactive TV. H.264 / MPEG-4 AVC, which is the turning point for video distribution over DSL and this new standard includes the following facts:

- Duplicates compression efficiency, reduces the bandwidth bandwidth required by the MPEG-2 standard for high-quality video transmission;

- Enables uploading more content over existing infrastructure because the same content after compressing with H.264 / MPEG AVC consumes less bandwidth;

- Reduces transmission costs because the same information comes twice as fast (of course because it is physically smaller and the quality is preserved);

- Reduces investment costs because the hardware required for H.264 / AVC is made up of commercial components that can be acquired in operations, ie for its realization it is not necessary to design special signal-processing hardware;
- Embedded Network Abstraction Layer (NAL) provides great flexibility in transmissions, whether packet networks or bitstream networks, enabling easy upgrades to existing MPEG-2 solutions;

- The number of DSL-search services to the distance that has not been able to offer more services and requirements so far has increased so that two standard video quality signals can be transmitted over one 1.5Mbps xDSL;

- More content can be uploaded to a wider area, i.e. up to a larger number of users, increasing the total number of available IPTVs. MPEG-2 can cover an area of about $810 \mathrm{~m} 2$ while H.264 / MPEG-4 AVC allow to cover an area of approximately $1440 \mathrm{~m} 2$;

- Greater coverage does not have to be achieved through the implementation of expensive amplifiers or the creation of new regional centers;

- The H.264 / MPEG-4 AVC codec can be implemented on commercial and industrial standardized equipment and there is no need for specially developed process equipment.

\section{QoS for Mobile Networks}

Integration of $2 \mathrm{G}$ (GSM), 2.5G (GPRS, EDGE), 3G (UMTS, CDMA2000), 3.5G (HSDPA), 4G, 4G advanced cellular networks with public, home, business as well as ad-hoc networks. Such architecture also implies the use of some new network elements such as servers and network passes for particular types of media. Servers should allow access to applications, while network passes provide the ability to connect to different types of networks, including access networks. Establishing such heterogeneous network architecture involves solving numerous problems such as: full user mobility, very fast handover, satisfactory service quality, security and tariffing. At a 5G environment, the blend of different wireless technologies and service providers that share an IP-based core network, will offer the possibility to the mobile devices of switching between providers and technologies, for maintaining a high level of Quality of Service (QoS).

By implementing the $5 \mathrm{G}$ mobile network apart from the goal of integrating mobile and fixed existing networks and services, as well as virtualizing infrastructure and manageability through software, it is essential to increase its speed, reliability and quality of service to its use in different segments and areas, from information distribution to management, how individual segments on the level of smart buildings or cities, so to the integral traffic control, production process, and so on. 


\subsection{Mobile and Cellular IP (MIP and CIP)}

In networks where routing is performed by IP protocol, each user has a unique address that determines his/her location as well as identification. The mobility problem of the user is solved by the Mobile IP Standard (MIP) that maintains the same IP address regardless of the current terminal location, which allows for a unique identification. MIP performs delivery of all packages to the mobile terminal in motion and determines the location of the user based on geographic coordinates. In analogous to mobile technology, such IP mobility can be seen as roaming. Access to the core of an IP based network can be achieved through fixed networks (PSTN, xDSL, Ethernet LAN) or wireless networks (GSM, GPRS, UMTS, WLAN).[2] Mobile IP represents a routing protocol that meets the following requirements:

- The IP addresses of mobile nodes should stay the same in order to provide services on higher layers;

- A mobile node should be available to communicate with IP-mobile and IPCorrespondents;

- Mobile nodes must be at the same level of security and reliability as fixed nodes.

There are two standards: MIPv4 and MIPv6 and they both solve the problem of macromobility. Within the 4G system, the MIPv6 standard is used to solve the problems of overloading and, at the same time it is guaranteeing performance using optimized routing (MIPv6 (w / RO)). Optimized routing means that the mobile node (MN-Mobile Node) directly informs its correspondents about the current Care of Address $(\mathrm{CoA})$ and receives packages independently of the HA (Home Agent) which was not the case under the MIPv4 standard. In addition to the mentioned optimized routing, there are situations where there is no optimized routing, so HA (MIPv6 (w / oRO)) is needed, while hierarchical addressing also includes MAP (mobility anchor point) within HMIPv6.

In the case of a quick handover, the path is further complicated by the tunneling of the packet from the previous access router (pARPrevious Access Router) to the new access router (nARNew Access Router). The problem of micro mobility within the MIPv4 standard is resolved by mobile IP. Mobile IP refers to mobility within a domain, micro mobility, while the MIP standard supports macro mobility. The universal component of cellular IP networks is a base station that serves as an access point that simultaneously routes IP packets and integrates cellular control functions contained in a mob interchange center (MSC) and a base station controller (BSC). Mobile
IP networks connect to the Internet over a network pass. Mobility between network passes is governed by the MIPv4 standard, while mobility within the access network is secured through cellular IP.

\subsection{QoS and Handover}

Providing QoS over a radio interface to a $4 \mathrm{G}$ network is based on the existence of multiple service classes. In this way, wireless network operators will be able to define their own class sets and make tariffs based on them. Handover can occur within a homogeneous system and is then referred to as a horizontal handover, while more of a problem is a handover between access technologies, user terminals or applications, i.e. hyper handover. Unlike horizontal handover, hyper handover involves high granularity in terms of QoS.

The problem with the telecommunication system is that there is no QoS support during handover. This practically means that when the terminal is moved from one station to another, packets arriving at the previous station will be either rejected or forwarded to the new base station but without QoS support. However, within the 4G system, using Packet tunneling enabled QoS support during hyper handover. It is possible to define three classes of handover: fast (for real-time services), smooth (handover) and unobtrusive handover (minimal interruption in service perception).

\section{Conclusion}

From the results obtained it can be concluded that VoIP traffic can serve best the Unsolicited Grant Service (UGS) during the service. The UGS service stream is designed for the traffic of constant transmission speed. The UGS stream service processes traffic generated by VoIP calls in the most optimal way. During video traffic analysis, as the number of nodes increases, the rtPS stream of services is better than the EU (Best Effort) stream service for average jitter. It has been confirmed that better service quality is achieved by service flows designed for specific applications. WiMAX technology can be considered the right technology for transferring voice over IP and video content, especially for mobile mobility and high throughput bandwidth. WiMAX can offer a satisfying quality of voice and video streaming services by choosing the right model without significant loss. To ensure the appropriate quality of VoIP and video services on WiMAX networks according to the practical part of the research, we conclude that certain steps need to be taken. First, it is essential to define the predominant purpose of the network, VoIP or video transmission, or the essential quality of both services. 
Subsequently, it is important to choose the appropriate codec, according to this study G.711 for VoIP and H.263 for video transmission. After that, it is important to choose the appropriate flow of services according to the specific network specification and the necessary parameters. There are clear benefits resulting from maintenance of QoS within the envisaged limits, which is to maintain the network and the connection itself stable. That's why we have a stable reception of data, speech and video. The network does not fail and there is no requirement for additional investments. Damage can occur on the network itself as overload, network failure, as a requirement for additional investments. However, even more damages may arise from the dissatisfaction of the service user if he does not have the service on the receiving side according to the appropriate standards. In most cases this damage will result in abandonment of the service provider.

WiMAX is a wireless digital communication system, known as IEEE 802.16, and it can provide broadband access to $50 \mathrm{~km}$ for fixed devices and 5 to $15 \mathrm{~km}$ for mobile stations. Mobile WiMAX today is a broadband wireless standard that enables the Quadruple Play service - data, speech, video, and mobility, using a single network. It provides complete mobility for end users who can use internet-based applications while traveling at speeds of around $120 \mathrm{~km} / \mathrm{h}$, which also means overlapping support. It also provides robust mobility and platform for accelerating network and service convergence. WiMAX is a technology close to the future that will meet the high demand for fast and wireless Internet access. Its speed, wide range of activities, ensures a good quality of service that will be cheaper than the previous technology. It is very likely that WiMAX will soon be inevitable in everyday use.
The benefits of WiMAX over $4 \mathrm{G}$ and $5 \mathrm{G}$ networks are that WiMAX implementation is far cheaper than the $4 \mathrm{G}$ and $5 \mathrm{G}$ network implementation, and WiMAX is an excellent choice for private mobile broadband wireless networks, which will be the first choice for rural grids. The most important advantages of the $4 \mathrm{G}$ network are that it enables work at a much higher speed of mobile users - at speeds up to 450 $\mathrm{km} / \mathrm{h}$, as well as offering better mobile power terminal technology - uses SC-FDMA technology for uplink, modulation technology that saves the battery life of mobile terminal.

\section{References}

[1]. Ferrag, M. A., Maglaras, L., Argyriou, A., Kosmanos, D., \& Janicke, H. (2018). Security for $4 G$ and $5 G$ cellular networks: A survey of existing authentication and privacy-preserving schemes. Journal of Network and Computer Applications, 101, 55-82.

[2]. Rahmanovic, A., \& Saracevic, M. (2017). The Significance of Using Ict in Telemetric Monitoring, Process Weighing and Control of The Coal. Southeast Europe Journal of Soft Computing, 6(1).

[3]. Rahmanovic A., \& Saracevic M. (2016). Use of GPS telemetry monitoring system in mining. MEST Journal, 5(2), 117-124.

[4]. Vogel, A., Kerhervé, B., Bochmann, G. V., \& Gecsei, J. (1994, October). Distributed multimedia applications and quality of service: a survey. In Proceedings of the 1994 conference of the Centre for Advanced Studies on Collaborative research ( $\mathrm{p}$. 71).

[5]. Multimedia Communications Forum (1995) Multimedia communications quality of service. Inc. MMCF/95-010, Approved Rev 1.0

[6]. WG, N. (2007, August). Wimax forum network architecture stage 2: architecture tenets, reference model and reference points, part 2. In WiMAX Forum, Wimax End-to-End Network Systems Architecture.

[7]. IEEE LAN/MAN Standards Committee. (2006). IEEE Standard for local and metropolitan area networks Part 16: Air interface for fixed and mobile broadband wireless access systems amendment 2: Physical and medium access control layers for combined fixed and mobile operation in licensed bands and corrigendum 1. IEEE Std 802.16 e-2005. 